\title{
Cost effectiveness of nurse led secondary prevention clinics for coronary heart disease in primary care: follow up of a randomised controlled trial
}

\author{
James P Raftery, Guiqing L Yao, Peter Murchie, Neil C Campbell, Lewis D Ritchie
}

\begin{abstract}
Objective To establish the cost effectiveness of nurse led secondary prevention clinics for coronary heart disease based on four years' follow up of a randomised controlled trial.

Design Cost effectiveness analysis.

Setting 19 general practices in north east Scotland.

Participants 1343 patients (673 in intervention group and 670 in control group, as originally randomised) aged under 80 years with a diagnosis of coronary heart disease but without terminal illness or dementia and not housebound.

Intervention Nurse led clinics to promote medical and lifestyle components of secondary prevention.

Main outcome measures Costs of clinics; overall costs to health service; and cost per life year and per quality adjusted life year (QALY) gained, expressed as incremental gain in intervention group compared with control group.

Results The cost of the intervention (clinics and drugs) was $£ 136$ (\$254; €195) per patient higher (1998-9 prices) in the intervention group, but the difference in other NHS costs, although lower for the intervention group, was not statistically significant. Overall, 28 fewer deaths occurred in the intervention group leading to a gain in mean life years per patient of 0.110 and of 0.124 QALYs. The incremental cost per life year saved was $£ 1236$ and that per QALY was $£ 1097$.

Conclusion Nurse led clinics for the secondary prevention of coronary heart disease in primary care seem to be cost effective compared with most interventions in health care, with the main gains in life years saved.
\end{abstract}

\section{Introduction}

Implementation of secondary coronary prevention in primary care is now widely advocated. Several lifestyle measures (smoking cessation, healthy diets, exercise) and medical treatments (antiplatelet agents, blood pressure lowering, lipid lowering, $\beta$ blockade, angiotensin converting enzyme inhibitors) can reduce the risk of coronary events and death in patients with established coronary disease. ${ }^{1}$ In the United Kingdom, general practitioners will be rewarded financially for achieving certain target standards. ${ }^{2}$ The problem remains, however, of putting these measures into practice.

Several mechanisms to improve secondary prevention have been evaluated, of which the most successful to date has been nurse led secondary prevention clinics. ${ }^{3-5}$ Between 1994 and 1995, we undertook one of the randomised trials of nurse led clinics and found them to improve implementation of secondary prevention and health related quality of life at one year. ${ }^{6}$ We followed up the participants after four years and found that the improvements in secondary prevention had translated into reduced total mortality. ${ }^{4}$ Running clinics, however, uses resources in primary care, especially nurses' time. The clinics incur further costs owing to the increased prescribing of secondary preventive drugs. ${ }^{6}{ }^{7}$ When compared with other strategies to improve secondary prevention, such as audit and feedback and opportunistic screening, general practitioners may view nurse led secondary prevention clinics as expensive and be reluctant to incur the costs unless they know that the clinics are cost effective.

Previous studies have evaluated the cost effectiveness of primary prevention clinics and found them to be relatively expensive in terms of cost per life year gained. ${ }^{8}$ We assessed the costs and cost effectiveness of nurse led secondary prevention clinics, as practised in our previous randomised trial. Alternative interventions we assess are based on that trial. The outcomes in the trial justified the cost effectiveness analysis in terms of cost per life year. We also present cost utility estimates-cost per quality adjusted life year (QALY). The time frame for our study was 4.7 years, the mean duration of patient follow up.

\section{Methods}

The methods used in the randomised trial are described elsewhere. ${ }^{467}$ Briefly, the trial was set in a stratified random sample of general practices in north east Scotland. Participants were a random sample of patients with a working diagnosis of coronary heart disease but without terminal illness or dementia and not housebound. Participants assigned to the intervention group (randomised by individual) were invited to attend a nurse led secondary prevention clinic at their general practice for one year. The clinic protocol included review of symptoms and risk factors, treatment, promotion of healthy behaviour (non-smoking, healthy diets, exercise), and secondary preventive medical treatment (aspirin, blood pressure control, lipid control). After the initial study year, we fed back the findings on uptake of secondary prevention and health status to practices which were free to decide their own policies on running clinics in future. Most of the practices (10) continued to run clinics, but opened them to all patients. The remaining nine practices stopped running clinics, but by four years' follow up six practices had restarted them.

Data, extracted from general practice case notes at baseline, one year, and four years, included participants' record of attendances at secondary prevention clinics, cardiovascular drugs, cardiovascular events (for example, myocardial infarction, coronary artery bypass grafting, percutaneous transluminal coronary 
Benefits (deaths, life years, and quality adjusted life years (QALYS)) and costs of nurse led secondary prevention clinics for coronary heart disease compared with control group

\begin{tabular}{|c|c|c|c|c|c|}
\hline Variable & Intervention group ( $n=673$ ) & $\begin{array}{c}\text { Control group } \\
(n=670)\end{array}$ & Difference $(95 \% \mathrm{Cl})$ & $P$ value & Comment \\
\hline No of deaths & 100 & 128 & 28 & 0.038 & \\
\hline Mean life years* & 4.590 & 4.483 & $0.110(0.012$ to 0.210$)$ & 0.028 & $\begin{array}{l}\text { As observed over mean } 4.7 \text { years } \\
\text { follow up }\end{array}$ \\
\hline Mean QALYs at end of follow up & 3.175 & 3.051 & 0.124 (0.059 to 0.189$)$ & $<0.001$ & Area under curve \\
\hline Costs of intervention $(£)^{*}$ & 1015 & 879 & 136 (56 to 216) & $<0.001$ & $t$ test \\
\hline Other NHS costs $(£)$ per patient & 2005 & 2238 & -233 (-766 to 299) & 0.390 & $t$ test \\
\hline Incremental cost per life year $(£)$ & - & - & 1236 (268 to 17983 ) & Not applicable & Box method ${ }^{15}$ \\
\hline Incremental cost per QALY $(£)$ & - & - & 1097 (298 to 3633) & Not applicable & Box method ${ }^{15}$ \\
\hline Incremental net benefit ( $($ ) & - & - & 3584 (3501 to 3667) & $<0.001$ & Using threshold of $£ 30000 / Q A L Y$ \\
\hline
\end{tabular}

Benefits discounted at $3.5 \%$ and costs discounted at 3.5\%. Intervention includes costs for clinic and increased prescribing per patient. ${ }^{*}$ Discounted over four years at $3.5 \%$.

angioplasty), and use of private health care. At the same intervals, we collected data by postal questionnaire on health related quality of life (SF-36). ${ }^{9}$ We obtained data on deaths (dates and causes of death), hospital admissions, and outpatient attendances (dates and diagnoses) from the Scottish Morbidity Records held by the Information and Statistics Division, NHS Scotland, and we linked these anonymously.

All analyses were by intention to treat. The perspective was societal, including both public and private health services. As most participants were older than working age (mean (SD) ages at baseline: intervention group 66.1 (8.2), control group 66.3 (8.2)), we ignored effects related to employment.

We constructed Kaplan-Meier survival curves for total mortality, life years, and QALYs, and analysed these curves using the log rank test. QALYs were estimated for each individual by weighting survival days by utility scores, derived from the SF-36 returns, leading to quality adjusted survival curves. Owing to the large volume of data with practically complete data, our statistical analysis was based on $t$ tests, particularly for the most costly items (cardiovascular drugs in primary care, inpatient admissions). It was not feasible for us to report on each type of item prescribed because of the large number of different items involved. The main difference related to the prescribing of statins. ${ }^{4}$

We calculated observed mean life years, discounted at $3.5 \%$ over four years as recommended by the National Institute for Clinical Excellence. ${ }^{10}$ Using a recently published algorithm, we converted data on health related quality of life (SF-36) to overall quality of life scores and we used these scores to calculate QALYs. ${ }^{11}$ Overall costs to society comprised the costs to primary care, NHS hospitals, and private hospitals. We included hospital admissions related to cardiovascular disease only. Statistical analysis was based on $t$ tests owing to the large volume of practically complete datasets, particularly for the most costly items (cardiovascular drugs in primary care, inpatient admissions).

The total cost of the intervention to primary care included running the clinics and increased prescribing. On the basis of an audit of nurse time during the first year of the study and interviews with nurses and general practitioners after four years, those patients who attended in the first year of the clinics were attributed two visits and patients who attended in any of the subsequent years were attributed one visit. We found that initial visits lasted up to one hour, but that subsequent ones were shorter. In this analysis we have assumed that each visit lasted one hour. The costs of clinic materials and training were included at year 1 . In the second, third, and fourth years it was assumed that the only cost incurred in running the clinics was nurse time, which we put at $£ 20$ (\$38; €29) per hour. ${ }^{12}$ Using the Scottish Drugs Tariff, we costed prescriptions for cardiovascular drugs on the basis of data at baseline, one year, and four years. ${ }^{13}$ Although we did not include the capital costs (the building) of the clinics, mainly because of the variety of ways the clinics were organised in the trial, we did explore in the sensitivity analysis the effects of doubling the running cost of the clinics. We calculated the mean cost per patient to primary care (comprising the cost of the clinic and additional prescribing), discounted at $3.5 \%$ over four years. ${ }^{10}$

We calculated the cost of admissions to NHS hospitals by assigning the appropriate unit cost per case based on specialty and hospital and deriving costs per patient and total cost by group. ${ }^{14}$ Outpatient costs were based on the number of attendances multiplied by the relevant hospital unit cost. Missing data on outpatients were imputed on the basis of the average ratio of outpatient attendances per admission for cardiovascular disease for surviving patients. As the share of overall costs accounted for by outpatients was small, the imputation of these missing values made little difference. We costed admissions to private hospitals using NHS unit cost by specialty. Again, as these costs accounted for a small percentage of overall costs, the effects of this assumption were minimal.

\section{Results}

Patients assigned to nurse led secondary prevention clinics for coronary heart disease showed significant improvements in all components at one year (aspirin, blood pressure, lipids, exercise, diet) except smoking. ${ }^{6}{ }^{7}$ At four years, these improvements were sustained except for exercise. ${ }^{4}$ By year 4 , improvements were shown in the control group and differences were no longer significant, but the earlier differences had translated into significantly fewer deaths in the intervention group: 100 out of 673 (cumulative death rate $14.5 \%$ ) compared with 128 out of 670 $(19.1 \%)$ in the control group $(\mathrm{P}=0.038){ }^{4}$ This improvement in survival remained statistically significant after adjusting for age, sex, general practice, and baseline secondary prevention. Thus the intervention group had 28 fewer deaths. Over the four year period this generated an incremental 0.110 life years per patient in the intervention group or 0.124 QALYs per patient (table).

\section{Costs to primary care and overall costs to society}

The only differences in cost to primary care per patient were the direct costs of the intervention, specifically attendances at the secondary prevention clinic and increased prescribing. The costs were $£ 136$ higher (1998-9 prices) in the intervention group (table).

We have previously reported that total hospital admissions were lower in the intervention group, but part of this difference was accounted for by admissions for non-cardiovascular diseases, where the causal relation with secondary prevention clinics is questionable. ${ }^{6}$ For this reason we considered alternative estimates of overall costs to society, one including all types of admissions, the 


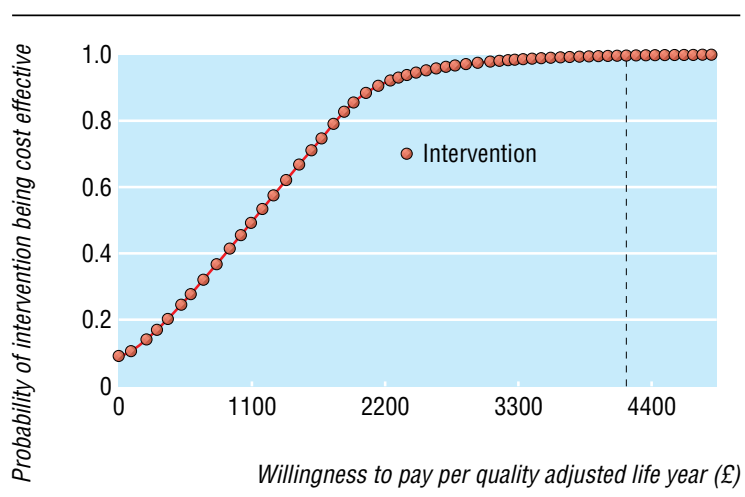

Cost effectiveness acceptability curve for nurse led clinics for secondary prevention of coronary heart disease

other confined to cardiovascular admissions. Although both estimates were lower in the intervention group, neither difference was statistically significant. Detailed analysis of costs of admissions to NHS hospitals, outpatient attendances, and admissions to private hospitals showed no statistically significant difference. When the costs to primary care were combined with hospital costs, the higher cost to primary care was offset by the lower hospital costs in the intervention group, such that the differences between intervention and control groups were insignificant. We therefore discuss costs related to primary care.

\section{Cost effectiveness}

The cost effectiveness analysis was based on the difference between groups in total cost to primary care (cost of the clinic and additional prescribing). The difference per patient was $£ 136$ (1998-9 prices). Combining the cost and outcome data gave an incremental cost per life year gained of $£ 1236$ and cost per QALY of $£ 1097$ (see table).

\section{Sensitivity analysis}

We used sensitivity analysis to explore increasing the cost difference only, on the basis that the benefits may have been underestimated owing to use of intention to treat analysis. We explored three changes in cost: (a) reducing the drug cost in the control arm to zero for all years, thus maximising the potential increment in drug cost attributable to the intervention (this increased the cost difference to around $£ 1000$ per patient, giving an incremental cost per QALY of around $£ 9000$ (£1000/0.11)); (b) increasing the cost of the secondary prevention clinic from the two visits in the first year and one visit in subsequent years to double that in the first year (but not in subsequent years as a high proportion of the control arm also attended these clinics; this increased the cost difference by around $£ 40$ in the first year only, increasing the total cost difference from $£ 136$ to $£ 176$ or by around $30 \%$ with a proportional effect on the cost per QALY); (c) combining both of these, which led to an incremental cost per QALY of just over $£ 9000$. We did not carry out tests for statistical significance on this analysis because the initial, smaller, cost difference was statistically significant.

The results of cost effectiveness analyses are increasingly presented in the form of cost effectiveness acceptability curves. By linking the probability that the intervention is cost effective at different levels of the willingness of the NHS to pay for an additional QALY, cost effectiveness acceptability curves substitute for confidence intervals. The figure shows the curve for the intervention group compared with the control group. This indicates a $70 \%$ probability of the intervention being cost effective if the NHS is willing to pay $£ 5000$ for an additional QALY, well below the putative NICE threshold of $£ 30000$. $^{16}$

\section{Discussion}

Nurse led clinics for the secondary prevention of coronary heart disease in primary care are relatively cost effective compared with the threshold of $£ 30000$ perceived by NICE. ${ }^{16}$ The intervention group gained a mean 0.110 life years and 0.124 QALYs compared with the control group. The incremental cost per life year saved was $£ 1236$ and that per QALY was $£ 1097$.

The findings of our original randomised trial that secondary prevention in terms of processes of care and prescribing is improved, accord with those of a systematic review of 12 randomised controlled trials of programmes for the management of chronic coronary heart disease. ${ }^{3}$ We showed that these benefits translated into reductions in total mortality in the medium term. ${ }^{4}$ The present study shows that the cost per life year gained, quality adjusted or otherwise, is less than $£ 1500$. The key difference reported was the increased $£ 136$ cost of the intervention to primary care, owing to attendances at the secondary prevention clinics and increased prescribing.

Although our evaluation was based on a well conducted randomised controlled trial we must consider limitations. ${ }^{17}$ Firstly, just over half the control group attended at least one secondary prevention clinic after the initial study year. ${ }^{4}$ Rather than compare secondary prevention clinics with unchanged usual care, we effectively evaluated the costs and benefits of having more patients attend secondary prevention clinics for longer. The total costs of running clinics to primary care (costs of the clinic and additional prescribing) will be higher than the cost difference between control and intervention groups in our study, as we used an intention to treat analysis, despite many patients in the control arm receiving the intervention in the period after the trial. Our estimates of cost effectiveness remain valid, however, as the benefits we found will also have been reduced by this cross over. The increase in both benefits and cost in practice depends on the pre-existing level of use of cardiovascular drugs, particularly statins, in the control group. Some of our data were incomplete, particularly attendances as hospital outpatients, but these had relatively little effect on overall costs to society-our data on high cost activities (for example, hospital admissions) and important outcomes, such as mortality, were almost complete. Where we made assumptions, such as for duration of appointments, we tended to overestimate the cost of the intervention.

Similarly, we ignored benefits if there were doubts that they could be attributable to the intervention, such as hospital admissions for non-cardiovascular conditions. We studied a random sample of general practices and patients, with good recruitment rates for both, so our sample should be representative of general practice at that time. We acknowledge, however, that changes may have occurred in practice since the study began in 1995 . The uptake that we reported for some secondary preventive drugs, especially statins, was lower than is likely in the current climate of national standards and incentives for general practitioners. Nevertheless, we found that the clinics improved uptake of secondary prevention by similar absolute amounts whatever the baseline levels, even for high uptake of activities at baseline such as blood pressure management and in practices with higher baseline levels of secondary prevention. ${ }^{6}{ }^{7}$ Newly recommended interventions, such as smoking cessation clinics and bupropion, may improve secondary prevention further but are unlikely to alter greatly the cost effectiveness as these changes are likely themselves to be highly cost effective. ${ }^{18}$

Our study is the first to examine the cost effectiveness of secondary prevention clinics in primary care. The most closely related cost effectiveness study, conducted in general practices in 
Belfast, was of personal health education to people with angina. ${ }^{19}$ An important difference is that in Belfast the intervention did not promote secondary preventive drug treatment, and drug costs were in fact reduced. Our findings, with increased drug costs, are more consistent with current recommendations and practice on secondary prevention and, indeed, provide a plausible explanation for the observed reduction in mortality. ${ }^{17}$ Despite this difference, the findings in Belfast were similar to ours: benefits to total mortality and health related quality of life were reported without a statistically significant extra cost in terms of total NHS resources. Clearly, whether or not the overall costs to society are statistically significant, practitioners in primary care are still faced with the real costs of providing the intervention, so we have gone further by calculating cost effectiveness on the basis of costs to primary care.

Other studies have evaluated the cost effectiveness of primary prevention clinics in primary care. ${ }^{8021}$ These analyses were limited because, firstly, benefits were slight and measured in terms of risk factors (so benefits to mortality had to be imputed) and, secondly, data on costs and savings to the health service were incomplete. Despite these limitations, some comparisons can be made: the running costs for clinics per patient are reasonably consistent across the trials; running costs for a practice population would, however, be much higher for primary prevention clinics because the target population would be much larger; the estimated cost effectiveness is much better for secondary prevention ( $£ 1236$ per life year gained) than for primary prevention (around $£ 30000$ per life year gained).

Compared with the wider range of health interventions, the cost effectiveness of secondary prevention clinics remains highly favourable. For example, interventions recommended by NICE have had a cost per QALY as high as $£ 39000 .{ }^{16}$ The incremental cost per QALY of under $£ 1000$ that we found was due to the relatively small increase in cost per patient of $£ 136$, mainly due to modest increases in drug use, even the relatively costly statins. This pattern, however, is consistent with other complex health service interventions, where incremental improvements in process outcomes are more likely to be achieved than wholesale changes. ${ }^{3}$ None the less, these relatively low increases in cost were linked to health gains that were considerable in terms of deaths, life years, and QALYs.

Contributors: JPR, PM, NCC, and LDR designed the study. PM and NCC collected the data.JPR, GLY, PM, and NCC analysed the data.JPR, GLY, PM, NCC, and LDR wrote the paper. JPR is guarantor.

Funding: The original trial and four year follow up study was funded by the Chief Scientist Office of the Scottish Executive. All researchers are independent of the Chief Scientist Office of the Scottish Executive.

Competing interests: None declared.

Ethical approval: The original trial and follow up study was approved by the Grampian research ethics committee.

1 Guideline 41. Secondary prevention of coronary heart disease following myocardial infarction. Edinburgh: Scottish Intercollegiate Guideline Network, 2000.

2 BMA/NHS Confederation. New GMS contract. www.bma.org.uk/ap.nsf/Content/ NewGMSContract (accessed 22 Jun 2004).

3 McAlister FA, Lawson FME, Teo KK, Armstrong PW. Randomised trials of secondary prevention programmes in coronary heart disease: systematic review. BMJ 2001;323:957-62

4 Murchie P, Campbell NC, Ritchie LD, Simpson JA, Thain J. Nurse led clinics for the secondary prevention of coronary heart disease: four year follow up of a randomised trial in primary care. BMJ 2003;326:84-7

5 Moher M, Yudkin P, Wright L, Turner R, Fuller A, Schofield T, et al. Cluster randomised controlled trial to compare three methods of promoting secondary prevention of coronary heart disease in primary care. BMJ 2001;322:1338-42.

6 Campbell NC, Thain J, Deans HG, Ritchie LD, Rawles JM, Squair JL. Secondary prevention clinics for coronary heart disease: randomised trial of effect on health. $B M J$ $1998 ; 316: 1434-7$

7 Campbell NC, Ritchie LD, Thain J, Deans HG, Rawles JM, Squair JL. Secondary prevention in coronary heart disease: a randomised trial of nurse-led clinics in primary care. Heart 1998;80:447-52

\section{What is already known on this topic}

Nurse led clinics for the secondary prevention of coronary heart disease improve implementation of secondary prevention and reduce mortality

Clinics require primary care resources and to increase prescribing

General practitioners may be unwilling to implement clinics unless they are shown to be cost effective

\section{What this study adds}

Nurse led secondary prevention clinics for coronary heart disease resulted in 28 fewer deaths and an increased cost to primary care of $£ 136$ per patient over four years

The gain in mean life years per patient was 0.110 and the gain in QALYs was 0.124 at an incremental cost of $£ 1236$ and $£ 1097$, respectively

Nurse led clinics for the secondary prevention of coronary heart disease in primary care seem to be cost effective compared with most interventions in health care

8 Wonderling D, McDermott C, Buxton M, Kinmonth A-L, Pike S, Thompson S, et al. Costs and cost effectiveness of cardiovascular screening and intervention: the British family heart study. BMJ 1996;312:1269-73.

9 Ware JE. SF-36 health survey-manual and interpretation guide. Boston: Nimrod, 1993.

10 National Institute for Clinical Excellence. Guide to the methods of technology appraisal. London: NICE, Apr 2004. www.nice.org.uk/page.aspx?o = 201974 (reference 0515).

11 Brazier J, Roberts J, Deverill M. The estimation of a preference-based measure of health from the SF-36.J Health Econ 2002;21:271-92.

12 Netten A, Curtis J. Unit costs of health and social care. Canterbury: Personal Social Services Research Unit, University of Kent, 2000.

13 Scottish drug tariff.Edinburgh: Scottish Executive Health Department, NHS in Scotland, 1998.

14 Scottish health service costs. Edinburgh: Information and Statistics Division, NHS in Scotland, 1999.

15 Polsky D, Glick HA, Wilke R, Schulman K. Confidence intervals for cost effectiveness ratios: a comparison of four methods. Health Econ 1997;6:243-52.

16 Raftery J. NICE: faster access to modern treatments? Analysis of guidance on health technologies BMJ 2001;323:1300-3.

17 Lancaster T. The benefits of nurse led secondary prevention clinics continued after 4 years. [Commentary.] Evid Based Med 2003;8:158.

18 National Institute for Clinical Excellence. Guidance on the use of nicotine replacement therapy (NRT) and bupropion for smoking cessation. Technology Appraisal Guidance No 39. London: NICE, 2002

19 O'Neill C, Normand C, Cupples M, McKnight ME. Cost effectiveness of personal health education in primary care for people with angina in the greater Belfast area of Northern Ireland. J Epidemiol Community Health 1996;50:538-40.

20 Langham S, Thorogood M, Normand C, Muir J, Jones L, Fowler G. Costs and cost effectiveness of health checks conducted by nurses in primary care: the Oxcheck study. BMJ 1996;312:1265-8.

21 Wonderling D, Langham S, Buxton M, Normand C, McDermott C. What can be concluded from the Oxcheck and British family heart studies: commentary on cost effectiveness analysis. BMJ 1996;312:1274-8.

(Accepted 17 December 2004)

doi $10.1136 /$ bmj.38342.665417.8F

Health Economics Facility, Health Services Management Centre, University of Birmingham, Birmingham B15 2RT

James P Raftery professor

Guiqing L Yao research fellow

Department of General Practice and Primary Care, University of Aberdeen,

Foresterhill Health Centre, Aberdeen AB25 2AY

Peter Murchie Cancer Research UK research training fellow in primary care oncology Neil C Campbell senior lecturer

Lewis D Ritchie Mackenzie professor of general practice

Correspondence to:J P Raftery j.p.raftery@bham.ac.uk

\section{Amendment}

This is version 2 of the paper. In this version the figure has been changed. 